\title{
Role of Echocardiography for Immediate Postoperative ICU Management after Tetralogy of Fallot Repair
}

\author{
Goverdhan D Puri
}

\begin{abstract}
How to cite this article: Puri GD. Role of Echocardiography for Immediate Postoperative ICU Management after Tetralogy of Fallot Repair. J Perioper Echocardiogr 2018;6(2):33-35.
\end{abstract}

\section{Source of support: Nil}

\section{Conflict of interest: None}

Tetralogy of Fallot (TOF) is the most common cyanotic congenital heart disease and its surgical repair also is the most commonly performed corrective surgery for cyanotic heart diseases. ${ }^{1}$ Perioperative echocardiography plays an indispensable role during surgery for TOF repair. Role of intraoperative transesophageal echocardiography (TEE) for TOF repair has already been reviewed previously. ${ }^{2}$ In this issue, Ganesan R et al. has reviewed a single center's experience regarding various common variations of TOF detected by perioperative TEE and has discussed about their relevance. Choudhary et al. has reported a rare echocardiographic combination of anomalous left coronary artery from the pulmonary artery (ALCAPA) and TOF.

Although postoperative course of most of the patients undergoing TOF repair is typically uneventful, a portion of patients can have complications like cardiac tamponade, elevated right ventricular (RV) pressure, right heart failure, low cardiac output syndrome, supraventricular arrhythmias and even pulmonary arterial hypertension. ${ }^{3}$ Apart from routine postoperative hemodynamic, laboratory-based and tissue perfusion monitors, echocardiography helps in accurate identification and management of the various complications encountered in intensive care unit (ICU).

Up to $25 \%$ of infants undergoing congenital heart surgery experience low cardiac output syndrome (LCOS) in the immediate postoperative period. ${ }^{4}$ Along with clinical and tissue perfusion parameters, echocardiography can estimate cardiac output by measuring left ventricular outflow tract (LVOT) diameter and LVOT velocity time integral (VTI) by 2D and pulsedwave Doppler method, respectively. The trend of cardiac

\footnotetext{
Professor and Head

Department of Anesthesia and Intensive Care, Postgraduate Institute of Medical Education and Research, Chandigarh, India

Corresponding Author: Goverdhan D Puri, Professor and Head, Department of Anesthesia and Intensive Care, Postgraduate Institute of Medical Education and Research, Chandigarh, India, e-mail: gdpuri007@gmail.com
}

output and response to therapy can be monitored by monitoring LVOT VTI serially. Normal aortic VTI has been seen to range in children from a mean of $13.8 \mathrm{~cm}$ in neonates to $25.1 \mathrm{~cm}$ in children $>17$ years of age. ${ }^{5}$

In patients having LCOS in the postoperative period, bedside echocardiography is useful in ruling out any significant residual lesions, like ventricular septal defect (VSD), right ventricular outflow tract obstruction (RVOTO). Residual VSD can be located anywhere in the septum, being most common in the superior portion of the patch ${ }^{6}$. It can be detected by $2 \mathrm{D}$ and color Doppler mapping of the ventricular septum and the patch. Evaluation of residual RVOTO consists of identifying the site of obstruction using color, pulsed-wave, and continuous-wave Doppler, from the parasternal long-axis and short-axis views or subcostal views. Late peaking of spectral Doppler waveform across the RVOT suggests dynamic obstruction within RV cavity, while mid-systolic peaking suggests residual valvar or supravalvar stenosis. However, the standard grading of severity of RVOTO based upon peak instantaneous gradient may not be valid here as the existent reference values are valid only in presence of normal cardiac output. ${ }^{6} 2 \mathrm{D}$, color and spectral Doppler assessment of the mediastinal portion of the pulmonary arteries (PA) can detect/rule out any residual stenosis at these locations.

RV dysfunction can complicate the immediate postoperative course after TOF repair and also can lead to LCOS, especially in patients who required RVOT incision and trans-annular patch (TAP) reconstruction. Limited information exists on the accuracy of the current guideline-recommended echocardiographic parameters for assessment of RV function in patients with repaired TOF. ${ }^{6}$ Nevertheless, a quantitative approach is preferred to the qualitative "eyeball" method." The utility of the nongeometric parameters, like RV isovolumic acceleration, Tei index, $\mathrm{RV} \mathrm{dP} / \mathrm{dt}$ are unclear in patients with repaired TOF. ${ }^{6}$ In repaired TOF patients, correlation of the tricuspid annular plane systolic excursion (TAPSE) and the RV fractional area change (FAC) with the cardiac magnetic resonance (CMR)-derived RV ejection fraction (EF) is weak and modest, respectively. ${ }^{6}$ TAPSE value in normal children ranges from a mean of $9.1 \mathrm{~mm}$ in neonates to $24.7 \mathrm{~mm}$ in 18-year-old subjects. ${ }^{8}$ 
Pulsed-wave tissue Doppler derived velocity of the longitudinal motion of the tricuspid annulus (RV S') $<11 \mathrm{~cm} / \mathrm{s}$ correlates well with CMR-derived impaired $\mathrm{RV}$ function in patients with repaired TOF. ${ }^{6}$ Role of Doppler and speckle-tracking-derived myocardial strain parameters in assessment of RV function after TOF repair remains to be determined by clinical research.

Presence of RV hypertrophy together with intraoperative insult can lead to restrictive RV physiology, which can also adversely affect the immediate postoperative course in these patients. Standard measures of $\mathrm{RV}$ diastolic function using pulsed-wave Doppler-derived trans-tricuspid flow velocities have not been validated in patients with repaired TOF. ${ }^{6}$ Instead, various surrogate measures like Doppler profile in the main pulmonary artery (including late diastolic antegrade flow), right atrial (RA) dilatation, hepatic venous flow reversal, and changes in the caliber of the inferior vena cava (IVC) with the respiratory cycle- are used for this purpose. ${ }^{6}$

Pulmonary overcirculation and pulmonary edema after TOF repair can complicate the immediate postoperative course after TOF repair, especially in patients having significant major aortopulmonary collateral arteries (MAPCAs). In patients having minimal/ no pulmonary regurgitation (PR) after TOF repair, due to pulmonary valve (PV)-sparing repair or monocusp reconstruction of pulmonary valve, the pulmonary overcirculation may be estimated by measuring the difference between RV and LV outputs by echocardiographyfuture validation studies can be planned to correlate the difference between LV and RV outputs after TOF repair with the preoperative angiography findings, intraoperative surgical findings and postoperative incidence of pulmonary edema or congestion.

Severe palmonary arterial hypertension $(\mathrm{PAH})$ following TOF repair has a prevalence of $1 \%$ and can lead to poor outcome. ${ }^{9}$ Peak PA systolic pressure is estimated by peak RV systolic pressure (in absence of a residual RVOT obstruction), which in turn is estimated using peak tricuspid regurgitation (TR) jet velocity using the modified Bernoulli equation: $4 \mathrm{v}^{2}+\mathrm{RA}$ pressure. RA pressure is obtained by transducing the central venous catheter, present almost universally in all cases in the immediate postoperative patient after TOF repair. In patients having transannular patch, mean PA pressure can be estimated from the peak pulmonary regurgitation (PR) jet velocity. Presence of raised PA pressure should lead to search for branch or peripheral PA stenosis, pulmonary vascular disease, pulmonary vein stenosis, left heart disease or presence of MAPCAs. If TR/PR jet is absent or inadequate for estimation of RV/PA pressure, findings like shift of interventricular septum towards LV or systolic flattening of interventricular septum indicate increased RV pressure and can be monitored serially to qualitatively assess the RV pressure relative to $\mathrm{LV}$ pressure. Residual RVOTO should be searched for in presence of elevated RV pressure. In the absence of PR, pulmonary acceleration time (PAT) can be used to assess and serially monitor the trend of PA pressure.

LV systolic dysfunction can occur after TOF repair leading to LCOS in postoperative period. The biplane area-length method should be used to calculate ejection fraction (EF) whenever feasible. ${ }^{6}$ Novel parameters like mitral annular plane systolic excursion (MAPSE), LV longitudinal strain, MAPSE/LV length (MAPSE/L) can also be used to quantify LV dysfunction. ${ }^{6,10}$

Apart from specific considerations after TOF repair, echocardiography is of enormous help in the immediate postoperative period in assessment and management of hemodynamic fluctuations, assessment of volume status, ruling out significant pericardial collection/tamponade.

Thus, not only in intraoperative period, but also echocardiography acts as a quintessential tool for the perioperative physician in management of TOF patients in the immediate postoperative period. In the absence of validation of values of various recommended quantitative echocardiographic parameters in TOF patients/in patients with congenital heart disease, qualitative assessment often acts as the guide for the postoperative physician. Future studies are required to validate/establish cutoff values for different echocardiographic parameters specifically for TOF patients to further help the ICU physicians in managing these, often critically ill, children for improved patient outcome.

\section{REFERENCES}

1. Rao PS. Consensus on timing of intervention for common congenital heart diseases: Part II - Cyanotic Heart Defects. Indian J Pediatr 2013;80: 663-74.

2. Puri GD, Raj R, Tacy TA. Transesophageal echocardiography for tetralogy of Fallot repair: What a perioperative physician needs to know?. J Perioper Echocardiogr 2014;2(2):51-57.

3. Perry J, Lanzberg M, Franklin W, Webb G, Chang AC. Cardiac intensive care of the adult with congenital heart disease: basic principles in the management of common problems.World J Pediatr Congenit Heart Surg 2011;2:430-444.

4. Hoffman TM, Wernovsky G, Atz AM, Kulik TJ, Nelson DP, Chang AC, Bailey JM, Akbary A, Kocsis JF, Kaczmarek R, Spray TL, Wessel DL. Efficacy and safety of milrinone in preventing low cardiac output syndrome in infants and children after corrective surgery for congenital heart disease. Circulation. 2003;107:996-1002. 
5. Pees C, Glagau E, Hauser J, Michel-Behnke I. Reference values of aortic flow velocity integral in 1193 healthy infants, children, and adolescents to quickly estimate cardiac stroke volume. Pediatr Cardiol 2013;34:1194-200.

6. Valente AM, Cook S, Festa P, Ko HH, Krishnamurthy R, Taylor AM, Warnes CA, Kreutzer J, Geva T.Multimodality imaging guidelines for patients with repaired tetralogy of Fallot: A report from the AmericanSociety of Echocardiography: developed in collaboration with the Society for Cardiovascular Magnetic Resonance and the Society for Pediatric Radiology.J Am Soc Echocardiogr 2014;27:111-41.

7. Puchalski MD, Williams RV, Askovich B, Minich LL, Mart C, Tani LY.Assessment of right ventricular size and function: echo versus magneticresonance imaging. Congenit Heart Dis 2007;2:27-31.
8. Koestenberger M, Ravekes W, Everett AD, Stueger HP, Heinzl B,Gamillscheg A, et al. Right ventricular function in infants, children andadolescents: reference values of the tricuspid annular plane systolicexcursion (TAPSE) in 640 healthy patients and calculation of $Z$ scorevalues. J Am Soc Echocardiogr 2009;22:715-9.

9. Roisman ML, Beller BM, O'Keefe JD. Irreversible pulmonary hypertensionafter correction of tetralogy of Fallot. Chest 1972;62:34-38.

10. Terada T, Mori K, Inoue M, Yasunobu H.Mitral annular plane systolic excursion/left ventricular length (MAPSE/L) as a simple index for assessing left ventricular longitudinal function in children. Echocardiography 2016;33:1703-1709. 